\title{
Fatal Myopericarditis in a Patient With Lupus Erythematosus Supported by Extracorporeal Membrane Oxygenation: A Case Report
}

\author{
Soo Yong Lee, M.D. ${ }^{1}$, ji Hye Park, M.D. ${ }^{2}$, Dong Hoon Shin, M.D. ${ }^{3}$, Taehwa Kim, M.D. ${ }^{4}$, Seungjin Lim, M.D. ${ }^{5}$ \\ ${ }^{1}$ Division of Cardiology, Department of Internal Medicine and Research Institute for Convergence of Biomedical Science and Technology, Pusan \\ National University Yangsan Hospital, Pusan National University School of Medicine, Yangsan, ${ }^{2}$ Division of Rheumatology, Department of \\ Internal Medicine, Daedong Hosipital, Busan, ${ }^{3}$ Department of Pathology, Pusan National University Yangsan Hospital, ${ }^{4}$ Division of Pulmonology, \\ Allergy and Critical Care Medicine, Department of Internal Medicine, Pusan National University Yangsan Hospital, ${ }^{5}$ Division of Infectious \\ Disease, Department of Internal Medicine, Pusan National University Yangsan Hospital, Yangsan, Korea
}

Systemic lupus erythematosus (SLE) may occur in any organ. In patients with SLE, myocarditis is extremely rare and potentially life-threatening. Herein, we report on a patient with lupus myocarditis, diagnosed by myocardial biopsy, immunologic tests, and clinical manifestations. Our findings suggest that securing time for diagnosis via extracorporeal membrane oxygenation and other intensive care is helpful for obtaining a good prognosis. (J Rheum Dis 2021;28:165-170)

Key Words. Extracorporeal membrane oxygenation, Myocarditis, Systemic lupus erythematosus

\section{INTRODUCTION}

The cardiac involvement of systemic lupus erythematosus (SLE) with refractory shock is rare and life-threatening $[1,2]$. Lupus myocarditis (LM) is clinically found in $3 \%$ $9 \%$ of SLE patients [3]. Herein, we report a challenging diagnosis, which was obtained by myocardial biopsy, and the treatment of a patient with SLE who presented with cardiogenic shock.

\section{CASE REPORT}

A 62-year-old female without any known disease was transferred to the emergency department due to fever for 10 days and persistent dyspnea; she had no medication history until symptoms developed and was admitted due to shortness of breath with right-sided pleural effusion 17 days previously (Figure 1A). Thoracentesis performed during the previous hospitalization revealed a lympho- cyte-dominant exudate (red blood cell [RBC] 2,000/ $\mu \mathrm{L}$, white blood cell [WBC] $1,440 / \mu \mathrm{L}$, lymphocyte $66 \%$, protein $5.3 \mathrm{~g} / \mathrm{dL}$, albumin $2.6 \mathrm{~g} / \mathrm{dL}$ [serum albumin 3.6 $\mathrm{g} / \mathrm{dL}$ ], lactate dehydrogenase [LDH] $152 \mathrm{IU} / \mathrm{L}$ [serum LDH 209 IU/L], and adenosine deaminase [ADA] of 43 $\mathrm{IU} / \mathrm{L})$. Due to the increased lymphocytes and ADA, the patient was suspected to have tuberculous pleurisy and the following medications were administered: anti-tubercular drugs (isoniazid, rifampin, ethambutol, and pyrazinamide) and cefepime for 10 days. However, she complained of dyspnea and fever when she visited our hospital. The patient was alert, and the peripheral oxygen saturation was $99 \%$ with nasal oxygen at $6 \mathrm{~L} / \mathrm{min}$. Her blood pressure was 100/60 $\mathrm{mmHg}$, pulse was 99 beats $/ \mathrm{min}$, and body temperature was $38.2^{\circ} \mathrm{C}$. Laboratory results at the emergency department are shown in Table 1 ; increased WBC, bicytopenia (RBC and platelet), elevated $\mathrm{C}$-reactive protein, and high sensitive troponin I. Chest computed tomography showed right dominant

\footnotetext{
Received : January 13, 2021, Revised : May 13, 2021, Accepted : May 15, 2021

Corresponding to : Seungjin Lim (D)http://orcid.org/0000-0001-7939-9744

Department of Internal Medicine, Pusan National University Yangsan Hospital, 20 Geumo-ro, Mulgeum-eup, Yangsan 50612, Korea. E-mail : babopm@naver.com
} 

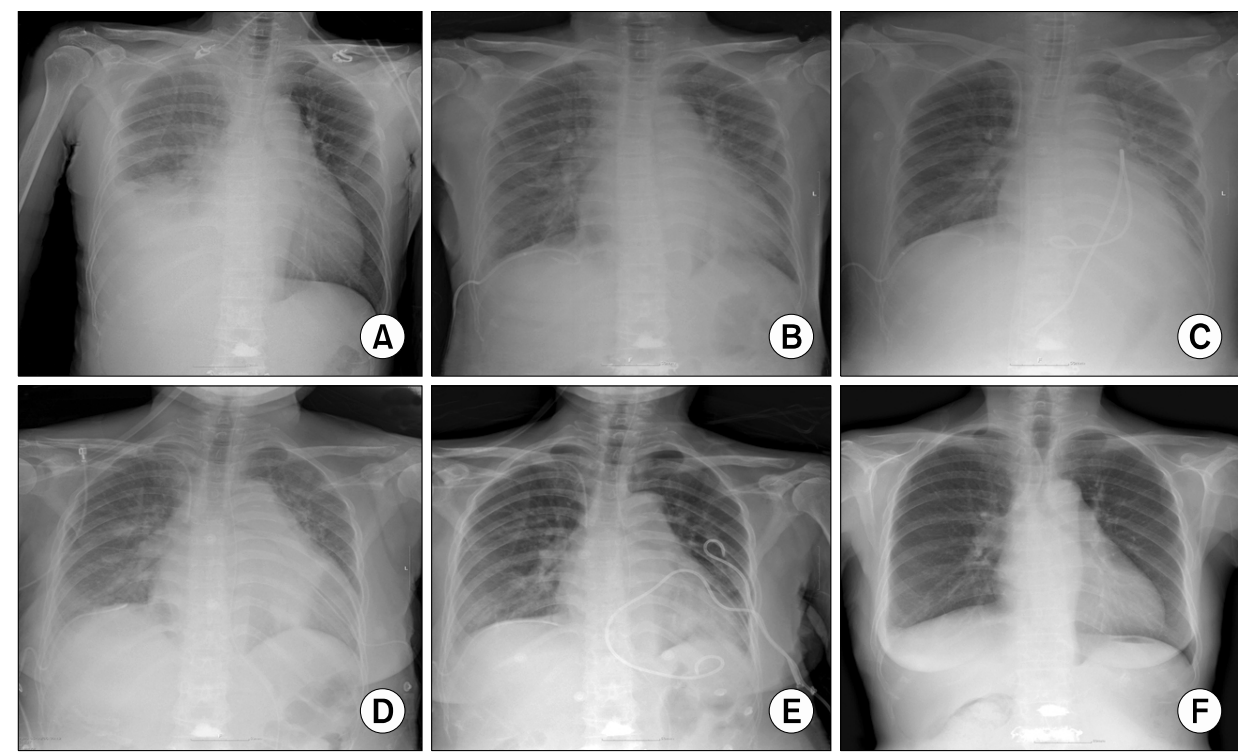

Figure 1. Chest $X$-rays of the patients over time. (A) At the time of previous hospital admission, 17 days before visit; (B) 1st day of hospitalization; (C) 3rd day of hospitalization (endomyocardial biopsy was performed); (D) 12th day of hospitalization (2nd pericardiocentesis was performed due to aggravated pericardial effusion); (E) 21st day of hospitalization ( 9 days after the restart of steroid therapy); (F) 49th day of hospitalization, at the time of discharge.

Table 1. Laboratory results of the case

\begin{tabular}{|c|c|c|}
\hline Tests & Results & Reference range \\
\hline $\mathrm{WBC}(/ \mu \mathrm{L})$ & 13,370 & $4,000 \sim 11,000$ \\
\hline Neutrophil $(/ \mu \mathrm{L})$ & 10,830 & $1,700 \sim 7,000$ \\
\hline Lymphocyte $(/ \mu \mathrm{L})$ & 530 & $1,000 \sim 4,000$ \\
\hline Hemoglobin (g/dL) & 10.9 & $13.5 \sim 17.5$ \\
\hline Platelet $(/ \mu \mathrm{L})$ & 85,000 & $140,000 \sim 400,000$ \\
\hline C-reactive protein (mg/dL) & 16.46 & $0 \sim 0.5$ \\
\hline Procalcitonin (ng/mL) & 6.084 & $<0.064$ \\
\hline D-dimer $(\mu \mathrm{g} / \mathrm{mL})$ & 4.76 & $<0.5$ \\
\hline High sensitive troponin I (pg/mL) & $1,327.67$ & $0 \sim 19.8$ \\
\hline Brain natriuretic peptide (pg/mL) & 304 & $0 \sim 100$ \\
\hline Rheumatoid factor (IU/mL) & $<10$ & $<10$ \\
\hline Lupus anticoagulant & Negative & Negative \\
\hline Anti-nuclear antibody & Positive 1: 320, speckled & Negative \\
\hline Anti-double stranded DNA IgG (IU/mL) & 49.9 & $<4.9$ \\
\hline ANCA & Negative & Negative \\
\hline Anti-SSA/Ro & Positive & Negative \\
\hline anti-La/SSB & Negative & Negative \\
\hline $\mathrm{C} 3 / \mathrm{C} 4(\mathrm{mg} / \mathrm{dL})$ & $77 / 27$ & $90 \sim 180 / 10 \sim 40$ \\
\hline CK (U/L)/CK-MB (ng/mL) & $77 / 7.7$ & $34 \sim 145 / 0.6 \sim 6.3$ \\
\hline $\mathrm{ESR}(\mathrm{mm} / \mathrm{hr})$ & 2 & $0 \sim 20$ \\
\hline C-reactive protein (mg/dL) & 16.46 & $<0.5$ \\
\hline EBV-PCR & Negative & Negative \\
\hline CMV-PCR & Negative & Negative \\
\hline Orientia tsutsugamushi & Negative & Negative \\
\hline Hantaan virus antibody & Negative & Negative \\
\hline Leptospira antibody & Negative & Negative \\
\hline TB-IGRA & Negative & Negative \\
\hline VDRL & Negative & Negative \\
\hline Anti-HIV & Negative & Negative \\
\hline
\end{tabular}

WBC: white blood cell, ANCA: antineutrophil cytoplasmic antibodies, C3: complement component 3, C4: complement component 4, CK: creatine kinase, CK-MB: creatinine kinase MB isoenzyme,erythrocyte sedimentation rate, EBV-PCR: Epstein-Barr virus-polymerase chain reaction, CMV-PCR: cytomegalovirus-polymerase chain reaction, TB-IGRA: tuberculous-interferon gamma release assay, VDRL: venereal disease research laboratory test, Anti-HIV: anti-human immunodeficiency virus. 
pleural effusion, and mild pericardial effusion.

Empiric antibiotics (piperacillin/tazobactam) were administered, and anti-tubercular medications were discontinued due to suspected side effects. While waiting for admission, she complained of chest discomfort and loss of consciousness. Her vital signs were blood pressure: $80 / 40 \mathrm{mmHg}$, heart rate: 130 beats/min, respiration rate: $35 / \mathrm{min}$, body temperature: $37.1^{\circ} \mathrm{C}$, and oxygen saturation: $80 \%$. Despite infusion of $2 \mathrm{~L}$ of crystalloids, hypotension persisted; high-dose inotropes and mechanical ventilation were started. Nonetheless, multi-organ failure progressed with metabolic acidosis and poor respiratory compensation (arterial blood gas analysis: $\mathrm{pH} 7.21$,
$\mathrm{PCO}_{2} 20 \mathrm{mmHg}, \mathrm{PO}_{2} 76 \mathrm{mmHg}$, base excess -19.9 , and $\left.\mathrm{HCO}_{3}-11.0 \mathrm{mM}\right)$. She underwent veno-arterial extracorporeal membrane oxygenation (VA-ECMO) following refractory shock, with continuous renal replacement therapy. Transthoracic echocardiography (TTE) demonstrated pericardial effusion compressing the right atrium and right ventricular (RV) wall and signs of a thickened left ventricular (LV) wall with severe systolic dysfunction (Figure 2A and 2B). VA-ECMO relieved the state of shock; however, we observed peripheral A wave variation of $>10 \mathrm{mmHg}$ and pulsus paradoxus. Pericardiocentesis was therefore performed, and $300 \mathrm{~mL}$ of serous fluid was drained. Following the disappearance of peripheral A
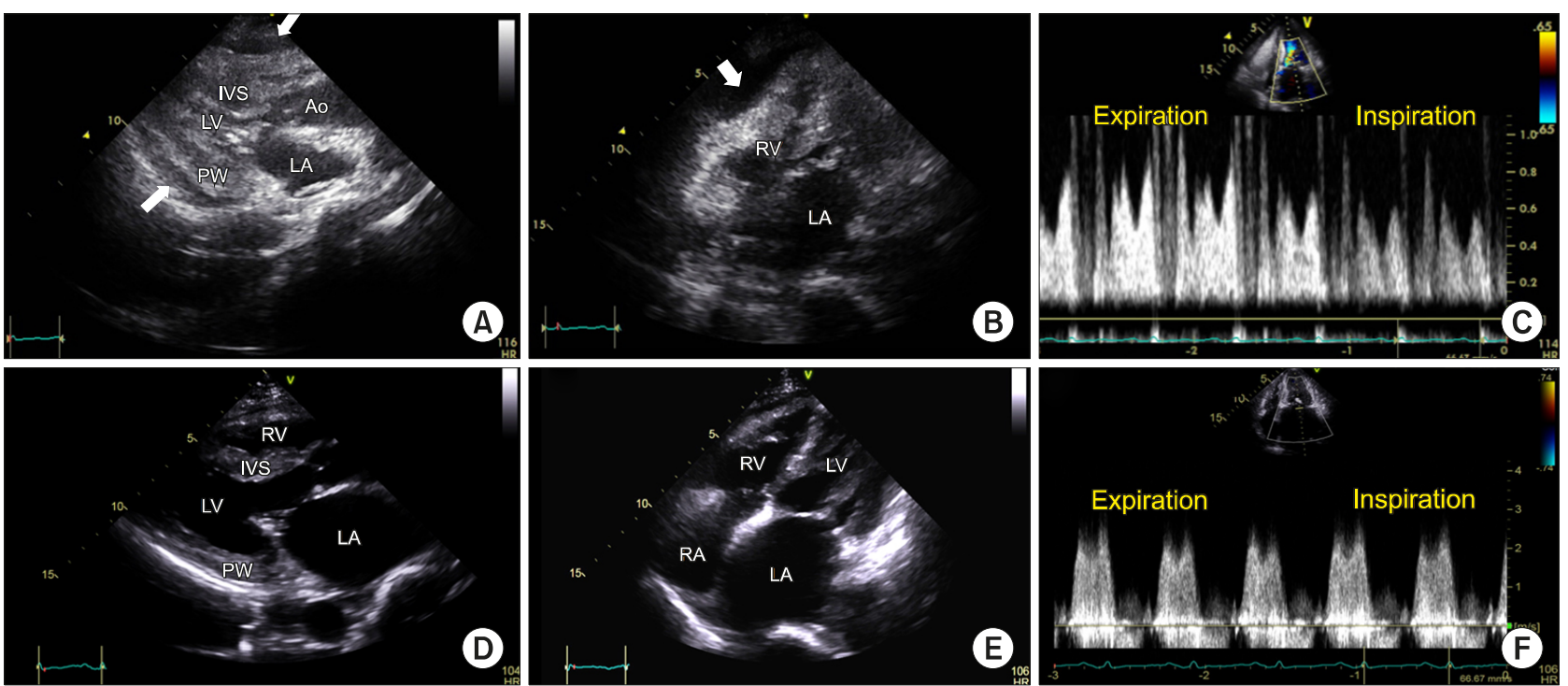

Figure 2. Echocardiogram. Still images $(A \sim C)$ have been captured after extracorporeal membrane oxygenation and before pericardiocentesis. (A) End diastole of the parasternal long-axis view shows a thickened LV wall with decreased LV cavity; (B) End diastole of the modified four-chamber view. Pericardial effusion compressing the right ventricular lateral wall is seen (arrows in $A$, B); (C) Mitral inflow pattern; the images $(D \sim F)$ have been captured 2 weeks after the steroid therapy. (D F) The ventricular wall thickness returned to the normal range compared with the initial echocardiogram (A). Mitral valvular calcification, thickening, doming, and elevated mean trans-mitral pressure gradient of $5 \mathrm{mmHg}$ suggested concomitant mild mitral stenosis. Ao: ascending aorta, IVS: interventricular septum, LA: left atrium, LV: left ventricular cavity, MV: mitral valve, PW: posterior wall, RV: right ventricle, RA: right atrium.
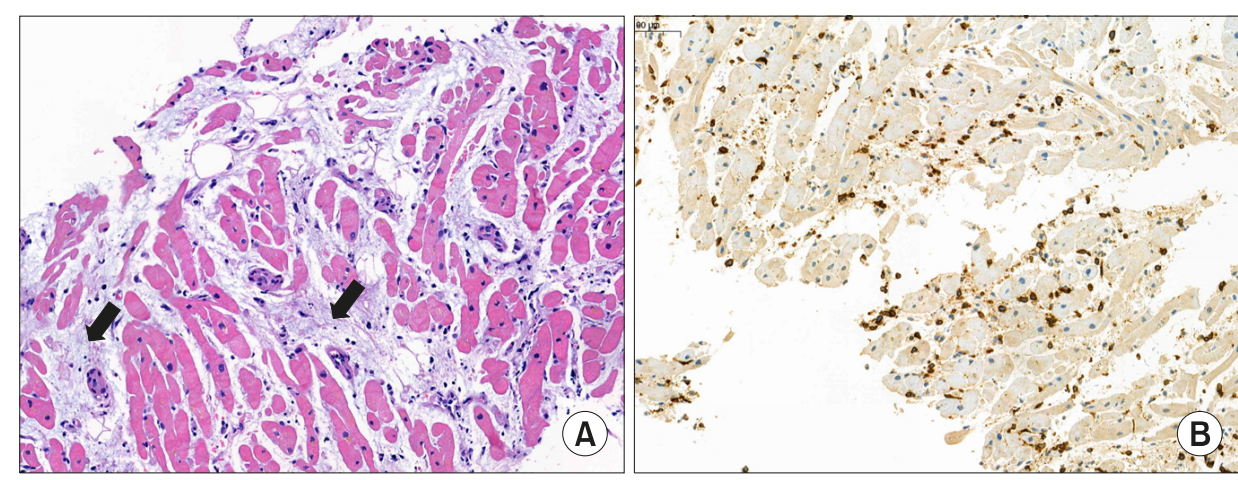

Figure 3. Pathologic findings. (A) Scattered lymphocytes and loss of myocardium replaced by progressive fibrosis (arrows) (H\&E stain, $\times 11$ ); (B) Immunohistochemistry of CD3. The diffusely scattered lymphocytes have been stained dominantly with CD3 $(\times 11)$. 
wave variation, the blood pressure increased from $86 / 72 / 76 \mathrm{mmHg}$ to $113 / 80 / 89 \mathrm{mmHg}$, and the heart rate decreased from 110 to 90 beats/min without vasopressor administration. Examination of the pericardial fluid demonstrated a total cell count of $542 / \mu \mathrm{L}$ (neutrophils [67.9\%] and lymphocytes [26.3\%]); LDH, $846 \mathrm{IU} / \mathrm{L}$; protein, $<3.0 \mathrm{~g} / \mathrm{dL}$; and ADA, $28 \mathrm{IU} / \mathrm{L}$; the sample tested negative for the tuberculous polymerase chain reaction.

Table 1 summarizes the laboratory results conducted to investigate the cause of myocarditis. Coronary angiography and RV endomyocardial biopsy (EMB) revealed no significant coronary artery occlusion. The biopsy revealed scattered lymphocytes with a loss of myocardium, consistent with myocarditis (Figure $3 \mathrm{~A}$ and $3 \mathrm{~B}$ ); findings suggestive of infiltrative cardiomyopathy were absent.

Since the patient's condition was critical, methylprednisolone (1 mg/kg, $70 \mathrm{mg}$ ) was empirically administered for 2 days after EMB on the 3rd to 4th day of hospitalization, while waiting for the biopsy results. The results of the biopsy suggested the possibility of idio- pathic myocarditis to be higher than that of amyloidosis or sarcoidosis; therefore, steroid therapy was discontinued after 2 days. Mechanical ventilation and ECMO were discontinued on the 7th and 9th days of hospitalization, respectively. On the 12th day of admission, she complained of chest discomfort accompanied by atrial fibrillation with a rapid ventricular rate. TTE was performed, and increased pericardial effusion was observed. Pericardiocentesis was performed, and $500 \mathrm{~mL}$ was drained. Since previous culture and microbiologic tests were negative, a non-infectious origin was considered.

We consulted the rheumatologist and performed additional tests. Profiles of autoantibodies are shown in Table 1; positive for anti-nuclear antibody (ANA), anti-double-stranded DNA antibody (anti-dsDNA antibody), and anti-Sjögren's-syndrome-related antigen A antibodies (anti-Ro52, anti-Ro60) and decreased levels of C3 and CH50. She was diagnosed with SLE on the 12th day of hospitalization; the patient fulfilled four of the 1997 American College of Rheumatology criteria (serositis

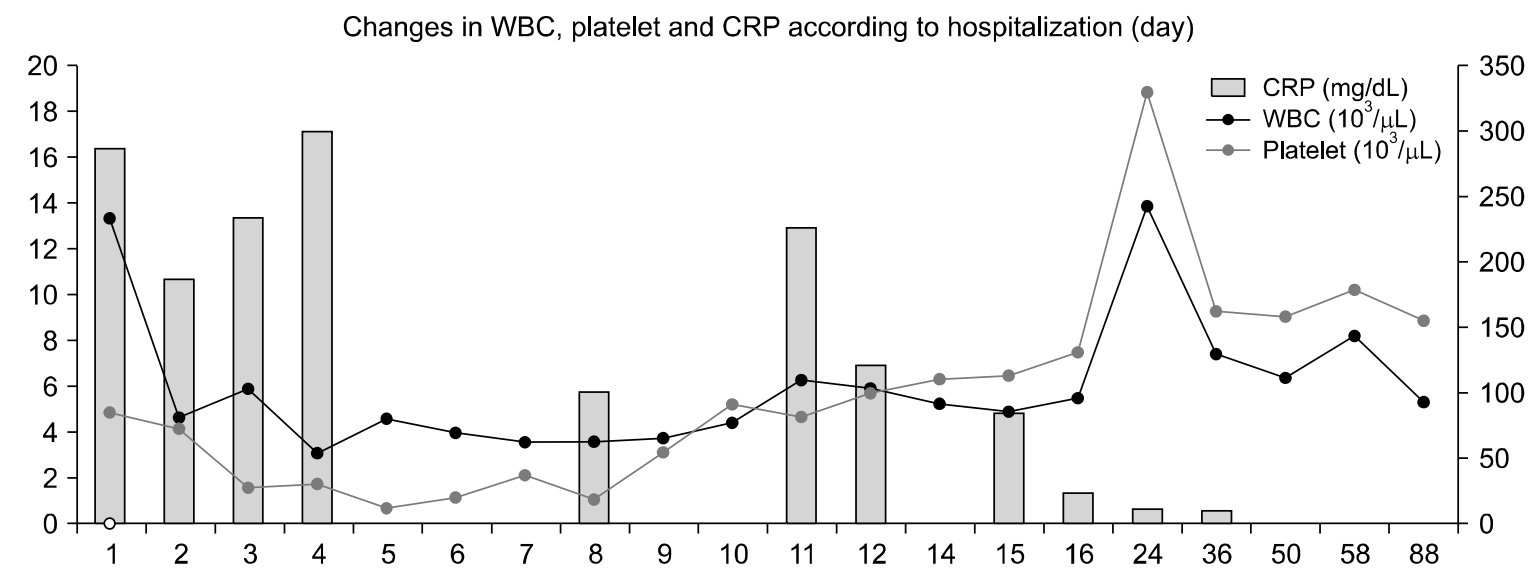

\begin{tabular}{|c|c|c|c|c|c|c|c|c|c|c|c|c|c|c|c|c|c|c|c|c|}
\hline Pericardiocentesis & 1st & & & & & & & & & & & 2nd & & & & & & & & \\
\hline \multicolumn{21}{|l|}{ EMB } \\
\hline \multicolumn{21}{|l|}{ ECMO } \\
\hline \multicolumn{21}{|l|}{ Mechanical ventilation } \\
\hline Steroid & & & \multicolumn{2}{|c|}{ MPD $70 \mathrm{mg}$} & & & & & & & & \multicolumn{4}{|c|}{ MPD $70 \mathrm{mg}$} & $50 \mathrm{mg}$ & $40 \mathrm{mg}$ & $30 \mathrm{mg}$ & $8 \mathrm{mg}$ & $4 \mathrm{mg}$ \\
\hline Hydroxychlorquine & & & & & & & & & & & & & & & & & & & \multicolumn{2}{|c|}{ HCQ $200 \mathrm{mg}$} \\
\hline Azathioprine & & & & & & & & & & & & & & & & & & & \multicolumn{2}{|c|}{ Azathioprine $25 \mathrm{mg}$} \\
\hline \multirow[t]{3}{*}{ Antibiotics } & \multicolumn{9}{|c|}{ Meropenem, vancomycin } & & & & & & & & & & & \\
\hline & & & & \multicolumn{13}{|c|}{ Levofloxacin } & & & & \\
\hline & & & & \multicolumn{7}{|c|}{ Doxycycline } & \multicolumn{6}{|c|}{ Cafepime } & & & & \\
\hline Day of hospitalization & 1 & 2 & 3 & 4 & 5 & 6 & 7 & 8 & 9 & 10 & 11 & 12 & 14 & 15 & 16 & 24 & 36 & 50 & 58 & 88 \\
\hline WBC $\left(10^{3} / \mu \mathrm{L}\right)$ & 13.37 & 4.61 & 5.96 & 3.07 & 4.62 & 3.95 & 3.63 & 3.58 & 3.73 & 4.44 & 6.36 & 5.94 & 5.24 & 4.85 & 5.47 & 14 & 7.38 & 6.4 & 8.24 & 5.32 \\
\hline Platelet $\left(10^{3} / \mu \mathrm{L}\right)$ & 85 & 73 & 28 & 31 & 13 & 20 & 37 & 19 & 56 & 92 & 81 & 100 & 111 & 114 & 131 & 332 & 162 & 159 & 180 & 156 \\
\hline $\mathrm{CRP}(\mathrm{mg} / \mathrm{dL})$ & 16.46 & 10.69 & 13.46 & 17.17 & & & & 5.8 & & & 12.96 & 6.94 & & 4.74 & 1.37 & 0.65 & 0.62 & 0.05 & 0.02 & 0.03 \\
\hline
\end{tabular}

Figure 4. Changes in laboratory findings and treatment according to hospitalization and outpatient follow-up periods. EMB: endomyocardial biopsy, ECMO: extracorporeal membrane oxygenation, MPD: Methylprednisolone, HCQ: hydroxychloroquine, WBC: white blood cell, CRP: C-reactive protein. 
[pleuritis], hematologic disorder, ANA positivity, and immunologic disorder) and seven of the 2012 Systemic Lupus International Collaborating Clinic criteria (serositis, leukopenia, thrombocytopenia, abnormal findings of ANA, anti-dsDNA, Anti-Sm, and low complement). Methylprednisolone ( $1 \mathrm{mg} / \mathrm{kg}, 70 \mathrm{mg})$ was restarted, and the patient's condition improved gradually; she was transferred to the general ward on the 23 rd day of hospitalization.

Echocardiography on the 14th day of hospitalization demonstrated normalization of the LV wall thickness and contractility. However, it revealed signs of mild rheumatic stenosis (Figure $2 \mathrm{D} \sim 2 \mathrm{~F}$ ). Cardiac magnetic resonance imaging was performed on the 37th day of hospitalization; myocardial inflammation and pericardial effusion had disappeared. However, diffuse late gadolinium enhancement of the entire pericardium suggested remnant pericarditis. The patient was discharged with methylprednisolone $(40 \mathrm{mg} /$ day $)$ on the 49 th day of hospitalization; the pleural effusion and cardiomegaly improved compared to previous chest radiographs (Figure 1B 1F). During follow-up, the dose of methylprednisolone was reduced to $4 \mathrm{mg}$ over 2 months with azathioprine (25 $\mathrm{mg}$ /day) and hydroxychloroquine ( $200 \mathrm{mg} /$ day). She has been doing well for the past 6 months without relapse of SLE (Figure 4) and has provided informed consent for publication of this report.

The study was approved by the Institutional Review Board of Pusan National University Yangsan Hospital (IRB No. 05-2021-001).

\section{DISCUSSION}

This case demonstrated fulminant myopericarditis caused by SLE. In this case, SLE was suspected only after clinical deterioration following discontinuation of steroid. Although proper laboratory tests were not performed at the beginning of hospitalization, the start of steroid therapy immediately after biopsy was considered to have helped improve the patient's condition. Clinical suspicion of autoimmune diseases, including SLE, should be considered, and early evaluation may improve prognosis without a relapse.

Cardiac involvement in SLE may involve the pericardium, myocardium, endocardium, heart valves, and coronary or pulmonary arteries [4]. Pericarditis is one of the most characteristic disease manifestation in SLE [5]. Nonetheless, cardiac tamponade is uncommon $(\sim 2 \%)$
[5]. LM reportedly occurs in 9\% of SLE patients [6]. There are a few reports on clinically apparent fulminant LM [7]; those on ECMO-supported cases are even rarer, and the prognosis in LM is dismal. A multi-center study in 2017 reported 29 cases of LM; three patients required ECMO support, and two died [6].

Most often, myocarditis results from viral infections; less commonly, specific forms of myocarditis may result from other pathogens, toxic or hypersensitivity-inducing drugs, giant-cell myocarditis, or sarcoidosis. EMB is the gold standard for diagnosing myocarditis [8]; however, it is rarely performed in the clinic. Most myocardial histopathology reports on SLE comprise postmortem data [8]; such reports reveal mononuclear cell infiltrates, perivascular inflammation or arteriopathy, and necrosis of the cardiomyocytes [7]. Our patient was relatively older and had a thickened myocardium. Thus, we expected the EMB to reveal signs of infiltrative cardiomyopathy. However, the pathology results indicated myocardial inflammation, necessitating the administration of anti-inflammatory therapy. EMB is not always required to diagnose LM. If the immunological tests and clinical findings strongly suggest SLE, EMB may not have been considered. However, it was helpful in excluding other diagnoses, and in the case of suspected myocarditis with cardiogenic shock, some studies recommended EMB to confirm myocarditis [9].

The timing of appropriate steroid administration in myocarditis is unclear. Steroid administration is not recommended in myocarditis caused by viral infection [10]. However, it is often difficult to rapidly diagnose the etiologies of myocarditis [10]. Thus, some clinicians initiate steroids with or without intravenous immunoglobulin when patients clinically decompensate despite advanced cardiopulmonary support in an effort to treat cryptogenic primary immune processes $[10,11]$. In this case, steroid was empirically administered just after EMB until pathology results were confirmed.

The treatment of LM has not been standardized and is based on clinical experience. Acute LM requires urgent clinical attention [4]. Most treatment modalities include high-dose corticosteroid administration as first-line therapy [6]. Some studies suggest high-dose intravenous therapy (e.g., methylprednisolone pulses of 1,000 mg/day), followed by high doses of an oral preparation (e.g., prednisolone, $1 \mathrm{mg} / \mathrm{kg} /$ day) for 1 to 2 weeks [4]. Moreover, immunosuppressive drugs, such as cyclophosphamide, mycophenolate mofetil, azathioprine, or cyclosporine, 
have also been used to manage LM [6]. Optimization of the treatment of underlying SLE and coexisting conditions, such as coronary artery disease, was suggested [4]. In our case, after the acute phase of LM, the patient was administered oral methylprednisolone, azathioprine, and hydroxychloroquine without worsening SLE or recurrence of myocarditis.

\section{SUMMARY}

VA-ECMO and pericardiocentesis could help treat fulminant myocarditis with cardiac tamponade due to SLE. It is important to consider rapid evaluation of immune-mediated myocarditis. Early administration of steroid therapy may help improve myopericarditis caused by SLE.

\section{CONFLICT OF INTEREST}

No potential conflict of interest relevant to this article was reported.

\section{AUTHOR CONTRIBUTIONS}

T.K. contributed to the study conception and design. Acquisition of data was performed by J.H.P. D.H.S. contributed to analysis and interpretation of data. First draft of manuscript was written by S.Y.L. and S.L. All authors read and approved the final manuscript.

\section{REFERENCES}

1. Chung JW, Joe DY, Park HJ, Kim HA, Park HS, Suh CH. Clinical characteristics of lupus myocarditis in Korea. Rheumatol Int 2008;28:275-80.

2. Chaudhari D, Madani MA, Balbissi Md KA, Paul TK. Lupus myocarditis presenting as life-threatening overt heart failure: a case report with review of cardiovascular manifestations of systemic lupus erythematosus. J La State Med Soc 2015;167:220-2.

3. Mohanty B, Sunder A. Lupus myocarditis-a rare case. J Family Med Prim Care 2020;9:4441-3.

4. Wijetunga M, Rockson S. Myocarditis in systemic lupus erythematosus. Am J Med 2002;113:419-23.

5. Doria A, Iaccarino L, Sarzi-Puttini P, Atzeni F, Turriel M, Petri M. Cardiac involvement in systemic lupus erythematosus. Lupus 2005; 14:683-6.

6. Thomas G, Cohen Aubart F, Chiche L, Haroche J, Hié M, Hervier B, et al. Lupus myocarditis: initial presentation and longterm outcomes in a multicentric series of 29 patients. J Rheumatol 2017;44:24-32.

7. Miner JJ, Kim AH. Cardiac manifestations of systemic lupus erythematosus. Rheum Dis Clin North Am 2014;40:51-60.

8. Gartshteyn Y, Tamargo M, Fleischer S, Kapoor T, Li J, Askanase A, et al. Endomyocardial biopsies in the diagnosis of myocardial involvement in systemic lupus erythematosus. Lupus 2020;29:199-204.

9. Kociol RD, Cooper LT, Fang JC, Moslehi JJ, Pang PS, Sabe MA, et al. Recognition and initial management of fulminant myocarditis: a scientific statement from the American Heart Association. Circulation 2020;141:e69-92.

10. Barrie M, McKnight L, Solanki P. Rapid resolution of acute fulminant myocarditis after IVIG and steroid treatment. Case Rep Crit Care 2012;2012:262815.

11. Saito T, Katayama H, Kodani E. Is steroid therapy really banned for lymphocytic myocarditis before excluding viral infection? Eur Heart J 2019;40:1014-5. 